MATHEUS MARTINS TEIXEIRA COTA

Estudos em Chamaecrista (Leguminosae) do Planalto de Diamantina, Minas Gerais: flora e novidades taxonômicas 
Matheus Martins Teixeira Cota

\section{Estudos em Chamaecrista (Leguminosae) do Planalto de Diamantina, Minas Gerais: flora e novidades taxonômicas}

Studies in Chamaecrista (Leguminosae) of Diamantina Plateau, Minas Gerais: flora and taxonomic novelties

Dissertação apresentada ao Instituto de Biociências da Universidade de São Paulo como parte dos requisitos para obtenção do título de Mestre em Ciências, na Área de Botânica.

Orientador: Prof. Dr. Renato de Mello-Silva

São Paulo 

Cota, Matheus Martins Teixeira

Estudos em Chamaecrista (Leguminosae) do Planalto de Diamantina,

Minas Gerais: flora e novidades taxonômicas.

$212 \mathrm{p}$.

Orientador: Renato de Mello-Silva

Dissertação (Mestrado) - Instituto de Biociências da Universidade de São Paulo. Departamento de Botânica.

1. Campos Rupestres 2. Florística 3. Cadeia do Espinhaço

\section{Comissão Julgadora:}

$\operatorname{Prof}(\mathrm{a}) . \operatorname{Dr}(\mathrm{a})$.

Prof(a). Dr(a).

Prof. Dr. Renato de Mello-Silva

Orientador 

"Ser mineiro é comer quieto o fim das palavras. Mineiro que é mineiro tem fome de sílaba e deve ser por isso que guarda dentro do peito poemas inteiros. Entre tantas letras embaralhadas, 0 vagão das ideias se perde e a coisa vira trem, ou o trem vira coisa.

É, o trem tá feio.

Ser mineiro é escutar no silêncio uma prosa bonita e musicar em sotaque frases curtas. $O$ mineiro não fala, ele canta com um sorriso tímido no canto da boca.

Nem todo mineiro é tímido, mas todo mineiro carrega o charme da timidez. Das bochechas coradas, do sorriso amarelo que ganha novas cores num piscar de olhos abertos, bem abertos.

Mineiro parece não gostar de elogio, mas gosta, pode apostar. Sempre retruca, mas cá dentro tá todo feliz.

São seus olhos. Ele diz.

Mineiro se esconde em suas montanhas, mas desmorona em abraço apertado. Chora água doce e se derrama em cachoeira.

Ser mineiro é fazer da cozinha a melhor parte da casa. Receber os amigos com mesa farta. Mineiro tem mesmo fome seja de letra ou de amor.

O mineiro não se apaixona "pelas" pessoas e, sim, "com" as pessoas. Ser mineiro é sentir as coisas sem dar nome. É se confundir entre dois ou três beijinhos quando conhece alguém.

São três pra casar.

Ser mineiro é passar a noite inteira em um ônibus e ainda não sair de Minas. As montanhas parecem continentes, mas fazem tudo parecer pertim.

Élogo ali.

Nunca confie em um "ali" de mineiro.

De resto, pode confiar. Seja nas reticências que ele não diz ou nos versos dos seus poemas inteiros.

Ser mineiro é saber que as melhores coisas da vida não são coisas."

Coisas que nenhum mineiro te contou - Luana Simonini 



\section{AGRADECIMENTOS}

"Agradecimento: sm. (agradecer+mento) 1 Ação ou efeito de agradecer. 2 Gratidão, reconhecimento. 3 Recompensa, remuneração."

Seguindo essa definição de agradecimento e sabendo que várias interpretações podem ser feitas disso, apresentarei alguns momentos que proporcionaram todo o desenrolar deste trabalho, pelos quais sou muito grato! E todo reconhecimento gera um pouco de culpa, pois nem sempre selecionamos todos os momentos para contar esse pedaço da história. Portanto, já começo me desculpando caso algum fato não apareça, pois às vezes eu mesmo não me lembro de ter os feito, perdão pelo lapso de memória! Evitarei dar nomes e priorizar momentos, sendo estes muito importantes para o desenvolvimento deste mestrado.

E todo começo inicia-se de um fim. O deste caso é quando termino minha graduação em Diamantina, Minas Gerais. Durante quase seis anos, tenho que agradecer tudo que passei por lá. Agradeço a todos que conheci, aos amigos, responsáveis por toda diversão e ajuda que precisei, às repúblicas, palco de muitas histórias inenarráveis, um salve especial à Babilônia (in memoriam), Camilla's house, Carandiru e SintAliga. Aos amigos, professores, técnicos e demais funcionários da Universidade Federal dos Vales do Jequitinhonha e Mucuri, que tornaram muito mais simples o desenrolar deste mestrado, fornecendo laboratórios, propiciando campos nos arredores e em Diamantina, e pela paciência pelo prolongado encerramento da graduação. À Diamantina, pelas belezas históricas, naturais, carnavais, pelos campos rupestres rodeados de cachoeira, por ser grande parte do cenário desse projeto. Esse cenário é muito bem descrito por Spix e Martius:

"De certo modo, a vegetação do Distrito Diamantino é a mais peculiar e bem formada flora dos campos que se observa no planalto. As entroncadas Liliáceas arbóreas, Velózias e

Barbacênias, são aqui mais abundantes do que nas outras partes de Minas, e são mesmo consideradas, pela gente do lugar, como indício da existência de diamante. Por entre as gramíneas peludas, verde-cinzentas, que, em grandes extensões, revestem as planícies deste Distrito, sobretudo são as Eriocauláceas que estão em 
grande número, com as suas umbelas de flores alvas pequeninas; Labiadas lanudas, numerosas compostas, Chamaecristas de folhas bonitinhas e flores amarelas..."

Antes mesmo de sair de Diamantina, já estava prestando à prova para tentar entrar no mestrado. Depois de algumas tentativas, venho para São Paulo fazer um estágio e logo em seguida já inicio a pós-graduação. Agradeço ao meu orientador, pelo qual aceitou trabalhar com as Chamaecristas do Planalto de Diamantina e pelo conhecimento que compartilhou comigo sobre as plantas. Desculpe-me, às vezes, pelo silêncio. Agradeço aos amigos que me deram abrigo, seja por poucos dias ou por alguns meses até que me instalei de vez em São Paulo. À Universidade de São Paulo e ao Instituto de Biociências por possibilitar o desenvolvimento deste trabalho. Ao CNPq pela bolsa concedida. E Caetano Veloso descreve exatamente o começo de uma vida em Sampa:

"E quem vem de outro sonho feliz de cidade/ Aprende depressa a chamar-te de realidade"

Durante quase três anos morando em São Paulo, trabalhei a dificuldade em lidar com mudanças fazendo o que tanto gosto, viajar. Primeiro vieram os campos realizados em diversas cidades nos arredores de Diamantina, lugar de parada certa. Agradeço aos amigos que me fizeram companhia durante essas viagens, em especial ao grupo de eriocaulólogos que me deram suporte a ir a lugares imprescindíveis. Em seguida, disciplinas realizadas na USP, UNICAMP e ESALQ e o exame de qualificação. Agradeço aos professores envolvidos, colegas e amigos, pelo convívio, apoio, conhecimento, pela disposição em me receber em suas casas, pelas histórias geradas durante as disciplinas, enfim por serem vocês! Logo em após, vieram as viagens aos herbários em Minas Gerais. Agradeço aos curadores de todos os herbários visitados, por todo apoio a mim concedido. Os congressos de Botânica, sempre importantes na comunicação e no aprendizado. Belo Horizonte, Salvador, Santos, Botucatu, agradeço à hospitalidade, aos amigos que estiveram envolvidos nessas viagens. E num susto, surge a viagem para New York. Agradeço ao meu pai, que pode me proporcionar essa experiência internacional. Às novaerenses, mais que conterrâneas, e aos novos amigos que participaram das aventuras na cidade que nunca dorme. 
E as viagens continuaram, não exatamente como programado, mas uma certa frequência de viagens à Minas Gerais foi necessária. E exatamente na mesma época, estar em São Paulo era preciso, visto a participação em monitorias e do próprio desenvolvimento do trabalho. Agradeço o apoio da minha família, do meu pai, da minha mãe e do meu irmão. Serei eternamente grato! Agradeço aos professores do Laboratório de Sistemática e Anatomia do Departamento de Botânica do IB/USP e demais professores do mesmo instituto, pelos ensinamentos, conversas e convivência. Aos amigos, colegas, e técnicos do Lab., agradeço às ajudas, às conversas, aos cafés, aos bolos na copa, ao aprendizado gerado, aos HappyHours above the waves. Vocês são demais! Durante isso, ainda visitei alguns herbários paulistas, baianos, curitibanos, cariocas e brasilienses. Agradeço aos amigos e curadores envolvidos que tornaram fáceis e divertidas as viagens.

Apesar de várias viagens, consegui conhecer um pouco de São Paulo. Virada Cultural, Vila Madalena, Vila Indiana, Liberdade, Centro, Anhangabaú, Butantã, Pinheiros, Morumbi, Bráz, Augusta, Paulista, Consolação, 25 de março, Ipiranga, São João, Beco do Batman, Galeria do Rock, Matilha Cultural, Ouvidor 63, Casa Amarela, Minhocão, Viaduto do Chá, Copan, Edifício Martinelli, Praça Pôr-do-Sol, Praça Roosevelt, Ibirapuera, Pinacoteca, MAC, OCA, Museu da Língua Portuguesa (luto), MASP, Instituto Tomie Ohtake, Sala São Paulo, Boteco Prato Do Dia, Espaço das Américas, SESC Interlagos, SESC Pinheiros, CCPC, Zé Presidente, Calefação Tropicaos, Venga Venga, Bixiga 70, Estúdio Lâmina, Trackers, festas, pontos turísticos, litoral norte, Ubatuba, metrô, espetáculos, feiras de rua, teatros, comédias, ocupações artísticas, pizza, culinária mundial, food-truck, pão com mortadela, mercadão, shows, enfim: diversidade, cultura(s). À São Paulo, agradeço a experiência. Por fim, agradeço a você, que de alguma forma me influenciou. Concordando ou não, me fez pensar sobre.

E termino como comecei, porém ainda não sei contar daqui em diante, pois ainda não os vivi (rsrs). Doutorado, consultorias, trabalho. Dúvidas, muitas. Portanto, Gratidão é a palavra que melhor define o agora. 

"...Chamaecrista, with a large number of herbaceous or suffrutescent species, readily propagating by seed, and many of them very abundant in individuals, is an exceedingly puzzling one to botanists."

George Bentham 



\section{ÍNDICE}

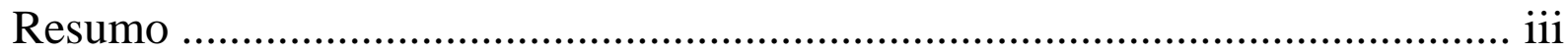

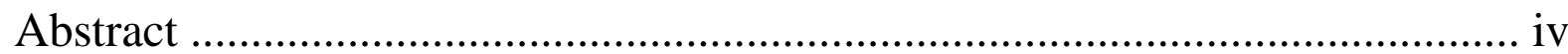

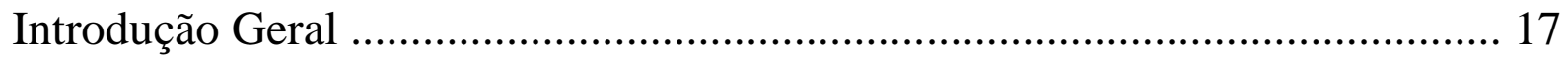

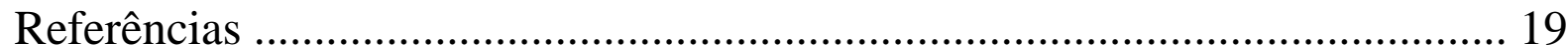

Capítulo 1

Chamaecrista (Leguminosae) do Planalto de Diamantina, Minas Gerais 21

Capítulo 2

Chamaecrista petiolata (Leguminosae, Caesalpinoideae), new species from Diamantina Plateau, Minas Gerais

Considerações finais 


\section{Resumo}

Floras são importantes no contexto moderno da biodiversidade, pois além de gerar informações iniciais para diversos estudos, promovem a melhor compreensão da diversidade de uma área. A crescente perda de habitats no bioma Cerrado, que inclui os campos rupestres e é um dos seis "hotspots" mundiais, alerta para a importância de estudos nesse bioma. A família Leguminosae possui alta representatividade nos campos rupestres e Chamaecrista é um de seus gêneros com grande número de espécies, muitas endêmicas. O gênero possui cerca de 330 espécies e duas características marcantes são as anteras pubescentes ao longo das suturas e nectários extraflorais. Apesar da grande importância das Leguminosas, estudos taxonômicos com essa família ainda são incipientes na região do Planalto de Diamantina. O presente trabalho teve como principal objetivo o estudo taxonômico das espécies de Chamaecrista do Planalto de Diamantina, Minas Gerais. O trabalho baseou-se no levantamento das espécies nos principais herbários com coleções do Planalto de Diamantina, em consultas bibliográficas e atividades de campo. Em sua forma final, a dissertação é dividida em dois capítulos, um referente à flora e outro referente à descrição de uma espécie nova. Neles, constam descrições das espécies, chave de identificação, mapas de distribuição geográfica, comentários taxonômicos e ilustrações. 


\section{Abstract}

Floras are important in the modern context of biodiversity, as well as generating initial information to several studies, promoting better understanding of the diversity of an area. The increasing loss of habitats in the Cerrado biome, which includes the rocky fields and is one of six "hotspots" worldwide, highlights the importance of studies in this biome. Leguminosae has high representation in rock fields and Chamaecrista is one of its genera with great number of species, many endemic. The genus has about 330 species and two striking features are the anthers pubescent along the sutures and extrafloral nectaries. Despite the great importance of Leguminosae, taxonomic studies of this family are still incipient in the Diamantina Plateau region. This study aimed to the taxonomic study of the species of Chamaecrista the Diamantina Plateau, Minas Gerais state. The work was based on the survey of the species in the main herbaria with collections from Diamantina Plateau, bibliographic consultations and field activities. In its final form, the dissertation is divided into two chapters, one relating to the flora and the other concerning the description of a new species. There are descriptions of species, key to species, geographic distribution maps, taxonomic comments and illustrations. 


\section{Introdução Geral}

O conhecimento das espécies de um local sempre gerou interesse, seja no âmbito local ou mundial, levando à criação de estratégias globais de conservação (Knapp 2008). Contudo, um dos maiores problemas enfrentados pelos taxonomistas é a delimitação das espécies, que pode levar a equívocos no conhecimento real da diversidade (Isaac et al. 2004, Knapp 2008). Além disso, espécies novas estão sendo descritas a cada novo estudo (Isaac et al. 2004, Knapp 2008, Rapini et al. 2008) e muitas espécies ditas endêmicas de um local têm se mostrado mais amplamente distribuídas (Gonella et al. 2014).

O campo rupestre é um ambiente de grande importância biológica e estudos florísticos nesse ambiente são necessários, pois existem muitas lacunas sobre a diversidade dessa fitofisionomia (Rapini et al. 2008). O Planalto de Diamantina deve ter sua flora estudada prioritariamente, já que a região abriga uma alta diversidade e tem sofrido com a antropização. O Planalto é o maior platô do Espinhaço mineiro e faz parte de um mosaico fitofisionômico e florístico influenciado por dois biomas, Mata Atlântica e Cerrado, gerando uma grande biodiversidade devido à concentração de ecótones (Gontijo 2008). Além disso, cerca de um quarto das espécies novas descritas no Brasil são provenientes de estudos realizados nos campos rupestres (Rapini et al. 2008).

Apesar da alta representatividade de Chamaecrista nos cerrados e nos campos rupestres (BFG 2015), e de um número razoável de estudos sistemáticos com o gênero (Irwin \& Rogers 1967, Irwin \& Barneby 1976, 1977, 1978, 1982, Fernandes \& Nunes 2005, Rando et al. 2013a), ainda são escassos os dados para a região do Planalto de Diamantina.

Dentro desse contexto, visamos contribuir com o conhecimento da diversidade de um gênero com alta representatividade na Cadeia do Espinhaço (Giulietti et al. 2000, César et al. 2006, Dutra et al. 2008, BFG 2015) que é uma região de extrema importância biológica, 
recentemente declarada Reserva da Biosfera pela UNESCO (Drummond et al. 2005). Esta dissertação, dividida em dois capítulos, apresenta estudos taxonômicos em Chamaecrista do Planalto de Diamantina em Minas Gerais.

O primeiro capítulo constitui uma flora, baseada nos meios clássicos de abordagens taxonômicas. Os objetivos principais foram a elaboração de descrições, ilustrações e chaves de identificação para as espécies. Além disso, apresentaram-se novidades taxonômicas, como a descrição de cinco espécies novas e novidades nomenclaturais. Este capítulo, em coautoria com Renato Mello-Silva, está formatado seguindo as normas da revista Acta Botanica Brasilica, na qual planejamos submeter o manuscrito.

O segundo capítulo apresenta a descrição de uma nova espécie, com ilustração, chave de identificação, além de discussões acerca de seu posicionamento sistemático e status de conservação. O manuscrito foi desenvolvido em coautoria com Juliana Gastaldello Rando e Renato Mello-Silva e submetido à revista Phytotaxa. 


\section{Referências}

Brazil Flora Group. 2015. Growing knowledge: an overview of Seed Plant diversity in Brazil. Rodriguesia 66(4): 1-29.

César EA, Juchum FS, Lewis GP. 2006. Lista preliminar da família Leguminosae na região Nordeste do Brasil - Série Repatriamento de dados do Herbário de Kew para a flora do Nordeste do Brasil. Vol.2. Royal Botanic Gardens. Kew.

Drummond GM, Martins CS, Machado ABM, Sebaio FA, Antonini Y. (Orgs.). 2005. Biodiversidade em Minas Gerais: um atlas para sua conservação. 2 ed. Fundação Biodiversitas. Belo Horizonte.

Dutra VF, Garcia FCP, Lima HC, Queiroz LP. 2008. Diversidade florística de Leguminosae Adans. nos Campos Rupestres. Megadiversidade 4: 117-125.

Giulietti AM, Harley RM, Queiroz LP, Wanderley MGL, Pirani JR. 2000. Caracterização e endemismos nos campos rupestres da Cadeia do Espinhaço. pp 311-318. In: Cavalcanti TB, Walter BMT (eds.). Tópicos atuais em Botânica. SBB/Embrapa. Brasília.

Gonella PM, Rivadavia F, Sano PT, Fleischmann A. 2014. Exhuming Saint-Hilaire: revision of the Drosera villosa complex (Droseraceae) supports 200 year-old neglected species concepts. Phytotaxa 156(1): 1-40.

Gontijo BM. 2008. Uma geografia para a Cadeia do Espinhaço. Megadiversidade. 4(1-2): 715 .

Irwin HS. 1964. Monographic studies in Cassia (Leguminosae: Casesalpinioideae). I. Section Xerocalyx. Memoirs of the New York Botanical Garden 12: 1-114.

Irwin HS, Barneby RC. 1982. The American Cassiinae. A synoptical revision of Leguminosae, tribe Cassieae, subtribe Cassiinae in New World. Memoirs of the New York Botanical Garden 35(1-2): 1-918.

Irwin HS, Barneby RC. 1976. Notes on the generic status of Chamaecrista Moench (Leguminosae- Caesalpinioideae). Brittonia 28: 28-36.

Irwin HS, Barneby RC. 1977. Monographic studies in Cassia (LeguminosaeCaesalpinioideae). IV. Supplementary notes on section Apoucouita Bentham. Brittonia 29: 277-290.

Irwin HS, Barneby RC. 1978. Monographic studies in Cassia (Leguminosae Caesalpinioideae) III. Sections Absus and Grimaldia. Memoirs of the New York Botanical Garden 30:1-277. 
Irwin HS, Rogers DJ. 1967. Monographic studies in Cassia (Leguminosae-Caesalpinioideae). II. A taximetric study of section Apoucouita. Memoirs of the New York Botanical Garden 16: 71-118.

Isaac NJB, Mallet J, Mace G. 2004. Taxonomic inflation: its influence on macroecology and conservation. Trends in Ecology and Evolution 19: 464-469.

Knapp S. 2008. A century of evolution: Ernst Mayr (1904-2005) species concepts and floras: what are species for? Biological Journal of the Linnean Society 95: 17-25.

Rapini R, Ribeiro PL, Lambert S, Pirani JR. 2008. A flora dos campos rupestres da Cadeia do Espinhaço. Megadiversidade 4: 15-23. 
Considerações Finais 
Há uma grande diversidade de espécies de Chamaecrista no Planalto de Diamantina, indicando a grande importância de estudos taxonômicos para esse gênero na região. A descoberta de seis espécies novas, a sinonimização de 26 nomes dentro de seis táxons e a redefinição de características diagnósticas, como os tricomas e as inflorescências, são exemplos disso. Apesar disso, são talvez necessárias mais coletas, pois nenhum táxon foi amostrado nos municípios de Augusto de Lima e Monjolos. Além disso, muitas localidades no Planalto não são de fácil acesso, dificultando a sua amostragem. São ainda perceptíveis problemas na delimitação de espécies, sendo preciso maiores esforços em estudos florísticos, ecológicos, biogeográficos e filogenéticos. 